\title{
Changes in lower extremity muscle function after 56 days of bed rest
}

\author{
B. Buehring, ${ }^{1,2}$ D. L. Belavý, ${ }^{1}$ I. Michaelis, ${ }^{1}$ U. Gast, ${ }^{1}$ D. Felsenberg, ${ }^{1}$ and J. Rittweger ${ }^{\mathbf{3}, 4}$ \\ ${ }^{1}$ Center for Muscle and Bone Research, Charité Universitätsmedizin Berlin, Free and Humboldt Universities, Berlin, \\ Germany; ${ }^{2}$ Cleveland Clinic, Cleveland, Ohio; ${ }^{3}$ Institute for Biomedical Research into Human Movement and Health, \\ Manchester Metropolitan University, United Kingdom; and ${ }^{4}$ Institute of Aerospace Medicine, German Aerospace Center \\ (DLR), Cologne, Germany
}

Submitted 4 November 2010; accepted in final form 21 April 2011

Buehring B, Belavý DL, Michaelis I, Gast U, Felsenberg D, Rittweger J. Changes in lower extremity muscle function after 56 days of bed rest. J Appl Physiol 111: 87-94, 2011. First published April 28, 2011; doi:10.1152/japplphysiol.01294.2010.-Preservation of muscle function, known to decline in microgravity and simulation (bed rest), is important for successful spaceflight missions. Hence, there is great interest in developing interventions to prevent musclefunction loss. In this study, 20 males underwent 56 days of bed rest. Ten volunteers were randomized to do resistive vibration exercise (RVE). The other 10 served as controls. RVE consisted of muscle contractions against resistance and concurrent whole-body vibration. Main outcome parameters were maximal isometric plantar-flexion force (IPFF), electromyography (EMG)/force ratio, as well as jumping power and height. Measurements were obtained before and after bed rest, including a morning and evening assessment on the first day of recovery from bed rest. IPFF $(-17.1 \%)$, jumping peak power $(-24.1 \%)$, and height $(-28.5 \%)$ declined $(P<0.05)$ in the control group. There was a trend to EMG/force ratio decrease $(-20 \% ; P=$ $0.051)$. RVE preserved IPFF and mitigated the decline of countermovement jump performance (peak power $-12.2 \%$; height $-14.2 \%$ ). In both groups, IPFF was reduced between the two measurements of the first day of reambulation. This study indicates that bed rest and countermeasure exercises differentially affect the various functions of skeletal muscle. Moreover, the time course during recovery needs to be considered more thoroughly in future studies, as IPFF declined not only with bed rest but also within the first day of reambulation. RVE was effective in maintaining IPFF but only mitigated the decline in jumping performance. More research is needed to develop countermeasures that maintain muscle strength as well as other muscle functions including power.

jump mechanography; muscle power; muscle force; EMG

A FUNCTIONING MUSCULOSKELETAL system is a vital component of successful, long-term human spaceflight missions. This is exemplified in the NASA Bioastronautics Roadmap (http:// bioastroroadmap.nasa.gov) (46), in which several key problems pertain to muscle, joints, and bone. A number of studies have examined the effects of microgravity on muscle, [for review, see $(1,21,25,47)]$ and have shown that microgravity leads to a decline in maximal muscle force, muscle crosssectional area (CSA), and volume. Muscle power (2, 4, 28, 30, 58) and neuromuscular properties, such as the neuromuscular drive, deteriorate $(26,27,34,36,48,63)$. Furthermore, changes in the connective tissues within the musculoskeletal system occur, leading, for example, to decreased tendon stiffness (39, $41,51)$. On a cellular level, microgravity leads to muscle fiber atrophy, suggested by muscle biopsy data and changes in

\footnotetext{
Address for reprint requests and other correspondence: J. Rittweger, Institute of Aerospace Medicine, German Aerospace Center (DLR), Linder Höhe 1, D-51147 Cologne, Germany (e-mail: joern.rittweger@dlr.de).
}

myofiber architecture and cell protein expression $(12,17,25$, 29, 30, 47, 49, 66, 67).

Apart from muscle force and size, the clinical importance of muscle power has become evident in recent studies in older adults. This data imply that muscle power correlates better with quality of life, activities of daily living, morbidity, and even mortality than muscle force or size $(6,16,22,33,62)$. Hence, it will be important not only to include the assessment of muscle power in routine muscle-function measurements but also to design training methods that are able to prevent both muscle force and power in the astronauts and deconditioned individuals. Bed rest studies in men (4) and women (68) have provided data suggesting that losses in muscle power are greater than losses in muscle force as a consequence of bed rest. If indeed muscle power is more affected by spaceflight and its ground-based analog models than muscle force, then this will have an impact on the development and selection of effective countermeasures.

Recently, vertical jump tests have been introduced in the experimental bed-rest setting as a promising measure of muscle power and neuromuscular function (28). As expected, there is a significant decline in jump power as a result of bed rest, which can be mitigated by resistive exercise (58). In addition to a marked loss in muscle power, we observed that muscle force not only declines during bed rest but also on the first day of reambulation after bed rest (12). It is therefore important to gather information on the dynamics of functional rehabilitation of different muscle parameters following bed rest.

Accordingly, it has been attempted in the Long-Term Bed Rest study in Toulouse to monitor changes in vertical jumping performance immediately after bed rest (58). However, due to technical difficulties, such data could be obtained only in a subset of volunteers. To ameliorate the dearth of information, we aimed in the current work to examine two different muscle functions, namely, maximal voluntary isometric plantar-flexion force (IPFF) and jump performance expressed in jump height and power before and after 56 days of simulated microgravity in a bed-rest setting. We were particularly interested in the immediate recovery phase of the first few days. We hypothesized that there would be a decline of these parameters in the first days before recovery of function started. Furthermore, we hypothesized that there would be a difference in the amount of function loss between muscle force and jump power. In addition, we also aimed to examine superficial electromyography (EMG) of the involved musculature to explore the neuromuscular system as a potential contributor.

The Berlin Bed Rest (BBR) study (54) was designed to test the efficacy of resistive vibration exercise (RVE) as a potential countermeasure against musculoskeletal deconditioning. This training combines resistive exercises of the upper and lower 
extremity with whole-body vibration (54), which has previously shown promising results in increasing muscle performance in athletes and in the elderly $(13,14,18,19,32,53)$. Moreover, vibration is thought to provide great strain rates, which seem to be specifically effective to induce an anabolic response in bone (43). Accordingly, vibration exercise has been proposed as a convenient exercise modality for bone (50). An additional rationale for the selection of vibration in the BBR study arises from the pronounced difficulty to maintain the calf musculature and in particular, the soleus muscle, during bed rest (4). Under the assumption that vibration elicits monosynaptic reflexes $(53,56)$, we argue that the musculature undergoes a large number of action potentials during a relatively short time (e.g., 25/s when the vibration frequency is set to $25 \mathrm{~Hz}$ ) and that this electrical activation is likely to maintain slow-twitch fiber integrity (64).

Within the published literature on musculoskeletal effects of bed rest, there is a relative paucity of information on the time course of these changes. However, any discrepancies in the time-course data for different subsystems may yield important information. Our hypothesis was that RVE would prevent loss of muscle force and power.

\section{METHODS}

The details of the methodology, including study design, RVE training, and participants, have been published elsewhere (54) and will only be summarized briefly here.

It is thought that the vibratory inputs stimulate additional muscle activity during contraction (60), via the muscle spindle system $(52,57$, 61 ), to produce a greater force of muscle contraction (42) and hence, stimulus for muscle maintenance and/or hypertrophy. We therefore decided to compare muscle force, muscle power, and electric muscle activity in the lower extremity in a nonexercising control group with a training group that performed RVE during bed rest. Our hypothesis was that RVE would prevent loss of muscle force and power.

Study design. The BBR was a randomized, controlled trial, approved by the ethics committee of the Charité Universitätsmedizin Berlin. All subjects gave their written informed consent. Twenty young and healthy males were randomized to either a RVE group or a control group. Both groups underwent 56 days of strict, horizontal bed rest. The data collection pertinent to what we report here was done within the 3 days prior to initiation of bed rest [abbreviated as baseline data collection (BDC)], as well as the 7 days after the end of the bed rest period [abbreviated as recovery data collection $(\mathrm{R}+)$ ]. The main focus of this work is the comparison of the BDC values with the data collected immediately after the end of bed rest on the morning of reambulation (data point " $\mathrm{R}+1 \mathrm{~m}$ ", taken between 7:30 AM and 11 $\mathrm{AM})$. Additional measurements were obtained in the evening of $\mathrm{R}+1$ ("R $+1 \mathrm{e}$ ", taken between 4:30 PM and $7 \mathrm{PM})$, on day $2(\mathrm{R}+2)$, day 4 $(\mathrm{R}+4)$, and day $7(\mathrm{R}+7)$ of recovery. In between the $\mathrm{R}+1 \mathrm{~m}$ and the $\mathrm{R}+1$ e testing sessions, subjects walked for $\sim 45 \mathrm{~min}$ at a very gentle pace in the park of the hospital under surveillance of the investigators, and no other muscular testing was carried out.

Participants. After thorough medical and psychological screening, 20 males, aged 20-45, without known musculoskeletal, hematologic, or mental diseases, were included after they signed informed consent.

Training. Individuals in the RVE group trained twice daily (morning and afternoon) except for Wednesday afternoon and on Sundays. Thus 11 exercise sessions were performed/wk. A purpose-built device that applies side-alternating vibration as well as an elastic spring system to create static resistance (Galileo Space, Novotec Medical, Pforzheim, Germany) was used. Participants performed four different types of exercise.
1) Squatting exercise: The knees were moved from $90^{\circ}$ to full extension in cycles under vibration exposure.

2) Heel raises: Under vibration exposure, the heels were elevated off the platform into ankle plantar flexion as long as the participants could sustain this (up to $40 \mathrm{~s}$ ). The knees were kept in almost complete extension during this exercise. This was done with the idea to strengthen the plantar flexors.

3) Toe raises: Under vibration exposure, the forefeet were elevated off the platform into ankle dorsiflexion (up to $40 \mathrm{~s}$ ). The knees were kept in complete extension during this exercise. This exercise was done to target the foot dorsiflexors so that forceful agonistantagonist cocontractions could be performed in the following exercise (see point 4).

4) Ten kicks: Under vibration exposure, 10 explosive pushes with 10 -s pauses of rest in between each were performed against the platform.

Morning sessions consisted of exercises 1-4 and afternoon sessions of exercises 1-3. During exercises 1 and 2, high resistive loads $(\sim 1.0-1.8 \times$ body wt) were applied to the legs and trunk via the elastic string system that was attached to the participants through shoulder straps. The loads were aimed to be $75-85 \%$ of the individual's one-repetition maximum. In the afternoon session, loads of $\sim 60-80 \%$ of that used in the morning session were applied. No resistive load was used during exercises 3 and 4 . Training intensity was progressed by increasing the vibration frequency when participants were able to perform the exercises for longer than $100 \mathrm{~s}$. Trained staff supervised all training sessions. The main hypothesis of the BBR study focused on the maintenance of bone mineral content in the distal tibia (55). Bearing in mind that muscle strength seems to be an important prerequisite for the maintenance of bone, the training regimen therefore attempted 1) to maintain muscle strength and 2) to elicit large muscle forces, e.g., by agonist-antagonist cocontractions. Thus although the exercises performed also targeted the major muscle groups affected in bed rest (e.g., plantar flexors and knee extensors), added exercises (e.g., explosive squats and toes raises) were performed that aimed to provide additional stimulus for bone formation.

Isometric plantar flexion. Maximal voluntary IPFF of the left leg was measured with a purpose-built dynamometer (see Fig. 1). It was designed for rapid use to enable repeated testing during the recovery phase, and it consisted of an adjustable frame to provide resistance to the thigh and a ground reaction-force platform (Leonardo, Novotec Medical). Participants performed three maximal IPFF contractions. Each contraction was to be maintained for $1 \mathrm{~s}$. The volunteers had as much time as they needed to completely recover between each effort. As the next step, we selected the contraction with the greatest force for analysis.

We then calculated $25 \%, 50 \%$, and $75 \%$ force values using the maximal contractions as $100 \%$. Following this, the volunteers performed plantar flexions at these force levels. They were instructed to get as close as possible to the predetermined force value and then keep this level for $2 \mathrm{~s}$.

The position of the foot on the force platform and seat height and the force to secure the knee in the dynamometer were recorded and used on the following measurements.

$E M G$. Superficial EMG of the calf muscles was recorded during IPFF. Bipolar electrodes (Dahlhausen, Cologne, Germany) were placed $3 \mathrm{~cm}$ apart from each other on the muscle belly of the medial and lateral gastrocnemius, as well as the soleus muscle, after shaving and skin abrasion. To ensure appropriate positioning of the electrodes, the distance from the middle of the electrode to the calcaneal insertion of the Achilles tendon was measured on the initial visit and used in subsequent tests. Ground electrodes were placed along the iliac crest. EMG signals were recorded with PowerLab and Chart software version 4.2 (both AD Instruments, Sydney, Australia) at a sampling rate of $2000 \mathrm{~Hz}$ with a 16-bit analog-to-digital converter. A band-pass filter $(10-500 \mathrm{~Hz})$ was applied to the signal. The root mean square (RMS) value of the EMG amplitude was used for analysis. RMS 


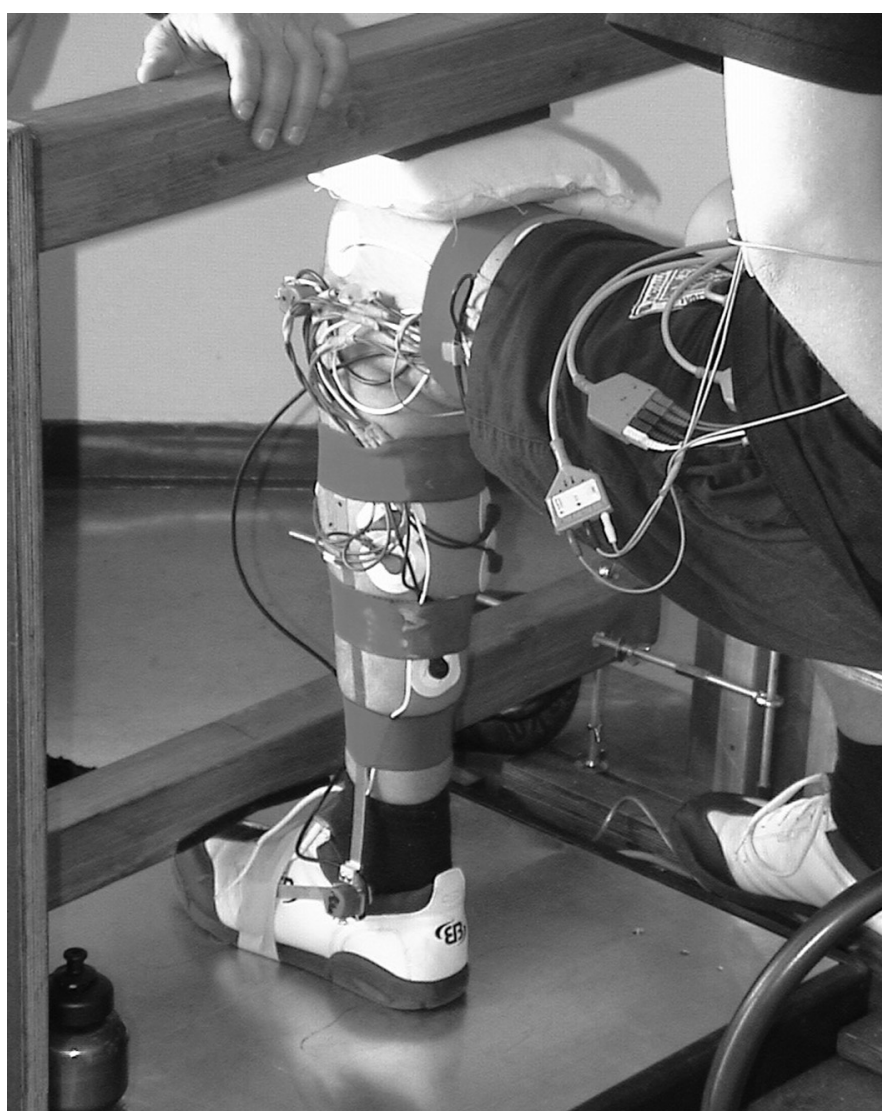

Fig. 1. The dynamometer used for the isometric plantar flexions. The dynamometer consisted of an adjustable frame (a horizontal bar held in place by two upright bars) and a ground-reaction platform. Participants were positioned with the foot in the middle of the platform and the knee joint in a $90^{\circ}$ angle.

values of the maximal plantar flexion were calculated from a 1-s time window, in which the participant was asked to keep the maximal force as constant as possible. For the submaximal 25\%, 50\%, and $75 \%$ contractions, a time window of $2 \mathrm{~s}$ was used. If the force varied more than $100 \mathrm{~N}$, the recording was marked as failed, and the participant repeated the contraction. Of interest were the RMS values of the maximal plantar flexion as well as the EMG-RMS-force relationship. We combined the RMS values of the individual muscles (soleus as well as lateral and medial gastrocnemius) by averaging the three values. The IPFF contraction with the highest amount of force of the BDC measurement served as the $100 \%$ force and EMG-RMS value. Next, percent values were calculated for submaximal $(25 \%, 50 \%$, $75 \%$ ) contractions in relation to the $100 \%$ RMS and force values. To examine changes in the EMG-force relationship, we chose the \% RMS-\% force gradient. This was obtained by first taking the mean of the three contractions \% RMS and \% force values at each intensity level and then dividing the \% RMS by \% force. Lastly, the mean gradient for each participant was calculated from the four different intensities $(25 \%, 50 \%, 75 \%, 100 \%)$ for each day before and after bed rest.

Jump mechanography. Jump mechanography has been described elsewhere $(58,59)$. In brief, it is performed on a ground reaction-force platform (Novotec Medical). The platform is divided into two separate plates. Ground-reaction data were analyzed as described in prior work (58), with self-built software (platon version 1.0.0.1, J. Rittweger).

In brief, force, velocity, peak power, and height are calculated using an established model $(15,23)$. After force is measured during quiet stance, body mass is calculated by dividing weight force by acceleration of gravity. Weight force then is used to calculate further parameters. Power - the product of force and velocity - is calculated with the measured force and the integration of acceleration over time. Integrating velocity over time results in displacement of the center of gravity or jump height. From this, we extracted the peak jump power during the push phase (commencement of countermovement to takeoff), specific to body wt $\left(\mathrm{P}_{\text {peak }}\right.$; in $\left.\mathrm{W} / \mathrm{kg}\right)$, the mean jump power, specific to body wt $\left(\mathrm{P}_{\text {mean }}\right.$; in $\left.\mathrm{W} / \mathrm{kg}\right)$, and maximal jump height $\left(\mathrm{H}_{\mathrm{jump}}\right.$; in $\left.\mathrm{cm}\right)$ for further analysis.

Statistical analysis. Group and time differences were tested with repeated measures ANOVA from BDC to $\mathrm{R}+2$. We chose these days, because we observed that both groups started to recover their muscle function by $\mathrm{R}+4$. We performed an a priori analysis of differences between $\mathrm{BDC}$ and $\mathrm{R}+1 \mathrm{~m}$ in addition to the repeated measure ANOVA. This was done using Student's $t$-test. Missing values were excluded from the analysis. The relation of \% EMG and \% force was graphically analyzed using linear regression. $\mathrm{R}^{2}$ values were used to express the degree of variability. Spearman's correlation coefficient was also calculated for the jump mechanography parameters at baseline (BDC; between absolute values) and also for the change between $\mathrm{BDC}$ and $\mathrm{R}+1 \mathrm{~m}$ (partial correlation coefficient controlling for study date). Statistical analysis was done using Microsoft Excel (Redmond, WA), SPSS (Chicago, IL; version 13), and JMP (SAS Institute, Cary $\mathrm{NC}$; version 8 ). We used an $\alpha$ level of 0.05 for statistical significance of findings.

\section{RESULTS}

Participants. Age and anthropometric data are presented in Table 1. The two groups did not differ in any of these parameters. All 20 volunteers finished the bed-rest phase and attended all follow-up appointments reported in this work. In total, 113 out of 120 jump experiments were performed as scheduled. However, because of either hardware or software failure, jump parameters were not obtained from one RVE participant on $\mathrm{R}+2$, one control participant on $\mathrm{R}+4$, and two $\mathrm{RVE}$, as well as three control participants, on $\mathrm{R}+7$.

$I P F F$. At baseline, maximal force values did not differ between groups $(P=0.825)$. We found a significant difference in the group and time analysis using repeated measures ANOVA $(P=0.0032)$. Following bed rest, IPFF was reduced by $17.1 \%$ in the control group at $\mathrm{R}+1 \mathrm{~m}$ from $1,136 \mathrm{~N}$ (SD 193 $\mathrm{N})$ at baseline to $943 \mathrm{~N}(\mathrm{SD} 222 \mathrm{~N} ; P=0.002)$. No such change was observed in the RVE group (BDC: $1,118 \mathrm{~N}, \mathrm{SD}$ $181 \mathrm{~N} ; \mathrm{R}+1 \mathrm{~m}: 1,138 \mathrm{~N}, \mathrm{SD} 160 \mathrm{~N} ; P=0.73)$. IPFF declined in both groups following $\mathrm{R}+1 \mathrm{~m}$. For the control group, such postreambulation decline was observed between $\mathrm{R}+1 \mathrm{~m}$ and $\mathrm{R}+1 \mathrm{e}$ only, and for the RVE group, there was also a significant decline on $\mathrm{R}+2$. Both groups started to regain IPFF by $\mathrm{R}+4$ (Fig. 2).

EMG and neuromuscular drive. We found a strong linear relationship of $\%$ force and \% RMS in both groups on BDC and $\mathrm{R}+1 \mathrm{~m}$, as shown in Fig. 3. The figure also displays the

Table 1. Demographic data of the two groups with 20 participants each during the enrollment visit

\begin{tabular}{lcccc}
\hline \hline Group & Age (Years) & Height $(\mathrm{cm})$ & Weight $(\mathrm{kg})$ & BMI $\left(\mathrm{kg} \cdot \mathrm{m}^{-2}\right)$ \\
\hline CTRL & $33.4(6.6)$ & $185(7)$ & $79.4(9.7)$ & $23.3(1.7)$ \\
RVE & $32.6(4.8)$ & $183(9)$ & $81.7(14.4)$ & $24.2(2.6)$ \\
\hline
\end{tabular}

Displayed are the mean values \pm SD. There were no significant differences $(P>0.1)$ between the two groups in any of the parameters. Reported are mean values \pm SD. BMI, body mass index; CTRL, control group; RVE, resistance vibrations exercise. 


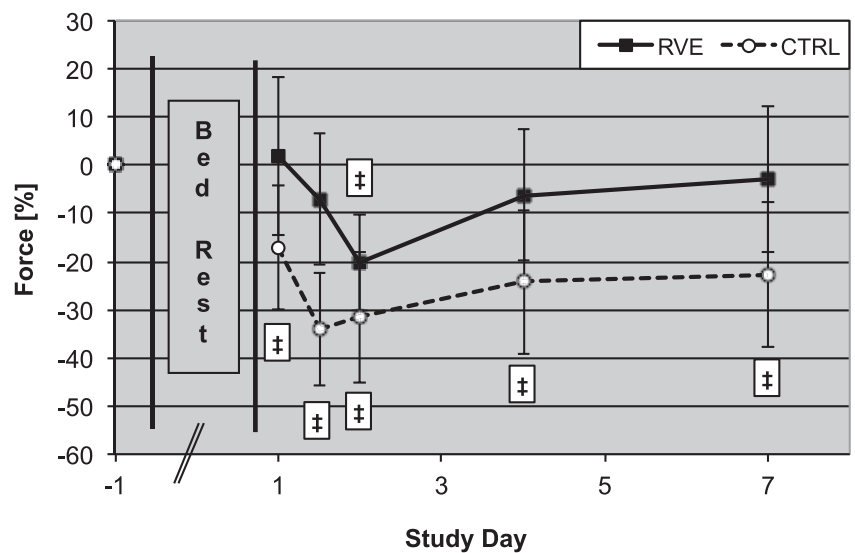

Fig. 2. Relative changes in maximal isometric plantar-flexion force in both groups from baseline data collection (BDC) to recovery data collection, day 7 $(\mathrm{R}+7)$. On data collected in the morning the 1 st day of recovery $(\mathrm{R}+1 \mathrm{~m})$, force was unchanged in resistive vibration exercise (RVE) but decreased in control group (CTRL). Interestingly, force declined in both groups between $\mathrm{R}+1 \mathrm{~m}$ and evening data collection the 1 st day of recovery $(\mathrm{R}+1 \mathrm{e})$. Lowest values were found on $R+1 e$ in the control and $R+2$ in the RVE group. Force started to improve after $\mathrm{R}+2$. There was a significant difference between groups in repeated measures analysis $(P<0.001)$. Changes within each group compared with baseline are represented by $\ddagger P<0.001$. Error bars represent SD. Study day -1 represents BDC. Days 1 and 1.5 represent $\mathrm{R}+1 \mathrm{~m}$ and $\mathrm{R}+1 \mathrm{e}$, respectively. Accordingly, study days 2,4 , and 7 represent $\mathrm{R}+2, \mathrm{R}+4$, and $\mathrm{R}+7$, respectively.

change in the \% RMS to \% force relationship before and after bed rest. We examined the slopes of the RMS-force relationship in both groups in an attempt to evaluate alterations in neuromuscular drive. The repeated measure ANOVA analysis did not yield a significant difference in the \% RMS/\% force gradient between the two groups over time $(P=0.117)$. The control group had a numerical increase of the slope after bed rest that trended toward significance $[P=0.051$; from 0.863 (SD 0.143) on BDC to 1.037 (SD 0.257) on $\mathrm{R}+1 \mathrm{~m}, 20 \%$ difference]. The slope was unchanged in the RVE group $[P=$ 0.615 ; from 0.858 (SD 0.196) to 0.901 (SD 0.315), a $5 \%$ difference]. Further testing using log-likelihood ratio tests showed that the intersubject variability of the $\% \mathrm{RMS} / \%$ force gradient was $2.47 \times$ higher after bed rest (on $\mathrm{R}+1 \mathrm{~m}$ ) than prior to bed rest $(P=0.0021)$ but that the training did not have a significant $(P=0.25)$ impact on this variability increase.

There was no significant change in the EMG amplitude during maximal plantar flexion in either group [control group: $0.414 \mathrm{mV}$ (SD 0.155) on BDC to $0.364 \mathrm{mV}$ (SD 0.098) on $\mathrm{R}+1 \mathrm{~m}, P=0.157$; RVE group: $0.426 \mathrm{mV}$ (SD 0.193) to 0.449 $\mathrm{mV}$ (SD 0.0813), $P=0.697$; data not shown].

Jump mechanography. Repeated measures ANOVA analyses showed significant differences between the two groups in the assessed time interval in both $\mathrm{P}_{\text {peak }}(P=0.007)$ and $\mathrm{H}_{\text {jump }}$ $(P=0.039)$. Interestingly, both groups had a significant loss in jump power and height after the bed-rest period, but the control group had a larger decline. On $\mathrm{R}+1 \mathrm{~m}, \mathrm{P}_{\text {peak }}$ decreased in the control group by $24.1 \%$ [from $43.37 \mathrm{~W} / \mathrm{kg}$ (SD 4.66) to 32.93 $\mathrm{W} / \mathrm{kg}$ (SD 7.21); $P=0.0004]$ compared with BCD. The RVE group lost $12.2 \%$ [from $43.6 \mathrm{~W} / \mathrm{kg}$ (SD 5.7) to $38.27 \mathrm{~W} / \mathrm{kg}$ (SD 4.7); $P<0.0001]$ in this time period. Changes in $\mathrm{P}_{\text {peak }}$ over time in both groups are depicted in Fig. 4. The magnitude of $\mathrm{H}_{\text {jump }}$ loss was similar to that of $\mathrm{P}_{\text {peak }}$ (Fig. 5). The decrease of $\mathrm{H}_{\text {jump }}$ in the control group was $28.5 \%$ [from $37.37 \mathrm{~cm}$ (SD 5.4) to $27.11 \mathrm{~cm}$ (SD 9.3); $P=0.0006$ ]. In the training group, the decrease was $14.2 \%$ [from $38.72 \mathrm{~cm}$ (SD 5) to $33.17 \mathrm{~cm}$ (SD 4.37); $P<0.0001$ ]. The results of $\mathrm{P}_{\text {mean }}$ were similar to those of $\mathrm{P}_{\text {peak }}$ and $\mathrm{H}_{\text {jump }}(12.3 \%$ loss in RVE, $P=0.0079 ; 33.1 \%$ in control, $P<0.0001$; repeated measures ANOVA, $P=0.003$; data not shown). There was a moderate correlation between $\mathrm{P}_{\text {mean }}$ and $\mathrm{P}_{\text {peak }}$ at $\mathrm{BDC}(P=0.005)$ and strong correlation $(P<0.00001)$ in the change between these parameters immediately after bed rest compared with baseline (Table 2).

In contrast to the IPPF results, $\mathrm{P}_{\text {peak }}$ and $\mathrm{H}_{\text {jump }}$ changed only minimally on $\mathrm{R}+1 \mathrm{e}$ and $\mathrm{R}+2$ in the control and $\mathrm{RVE}$ group. Improvements in $\mathrm{P}_{\text {peak }}$ and $\mathrm{H}_{\text {jump }}$ were seen on $\mathrm{R}+4$ in the controls but not in the RVE group.

When comparing the relative changes in IPFF with those of the countermovement jump experiments, the loss of $\mathrm{P}_{\text {peak }}$ and $\mathrm{H}_{\text {jump }}$ appeared larger than the IPFF in both groups. Statistically, however, only the differences in the RVE group were significant (IPFF vs. $\mathrm{P}_{\text {peak }}: P=0.014$; IPFF vs. $\mathrm{H}_{\text {jump }}: P=$ 0.0044 ) but not in the control group (IPFF vs. $P_{\text {peak }}: P=0.135$; IPFF vs. $\mathrm{H}_{\text {jump }}: P=0.178$ ).

\section{DISCUSSION}

The main findings of the current study were a significant decline in plantar-flexor muscle force and $\mathrm{P}_{\text {peak }}$ in the control group, with the loss in jump parameters nonsignificantly greater in magnitude than the decrease in IPFF (24\% vs. $17 \%)$. The EMG-force gradient in these individuals showed an in-

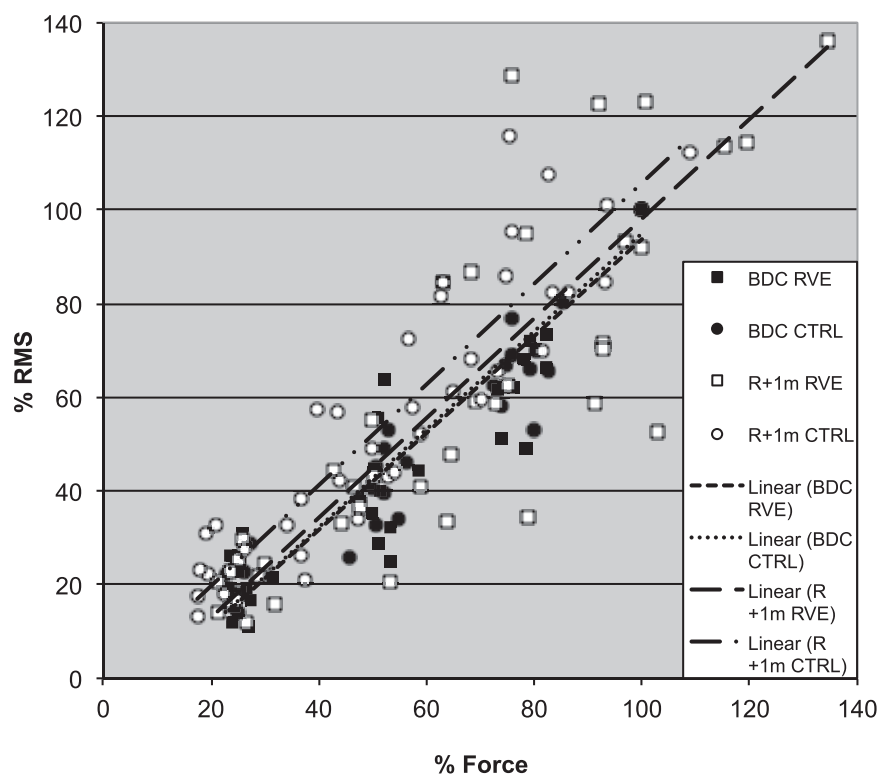

Fig. 3. \% Root mean square (RMS)-\% force relationship. To examine changes in neuromuscular drive before and after bed rest in the 2 groups, electromyography activity (expressed in \% RMS values) and force were plotted against each other. There was a linear relationship of RMS of the calf muscles and plantar-flexion force in both groups before and after bed rest. $\mathrm{R}^{2}$ values for the control group were 0.95 on BDC and 0.83 on $\mathrm{R}+1 \mathrm{~m}$. The RVE group had values of 0.91 for BDC and 0.67 for $\mathrm{R}+1 \mathrm{~m}$ (all $P<0.001$ ). In the control group, participants had higher \% RMS values on $\mathrm{R}+1 \mathrm{~m}$ for the same amount of \% force compared with BDC. In the RVE group, the regression line was also shifted in this direction but not as pronounced. This suggests a loss in neuromuscular drive in both groups that is larger in the control group. Statistical analysis of the \% RMS/\% force gradients showed a trend toward significance $(P<0.051)$ in the control group and no significant change in the RVE group $(P=0.615)$. Linear, linear regression curve. 


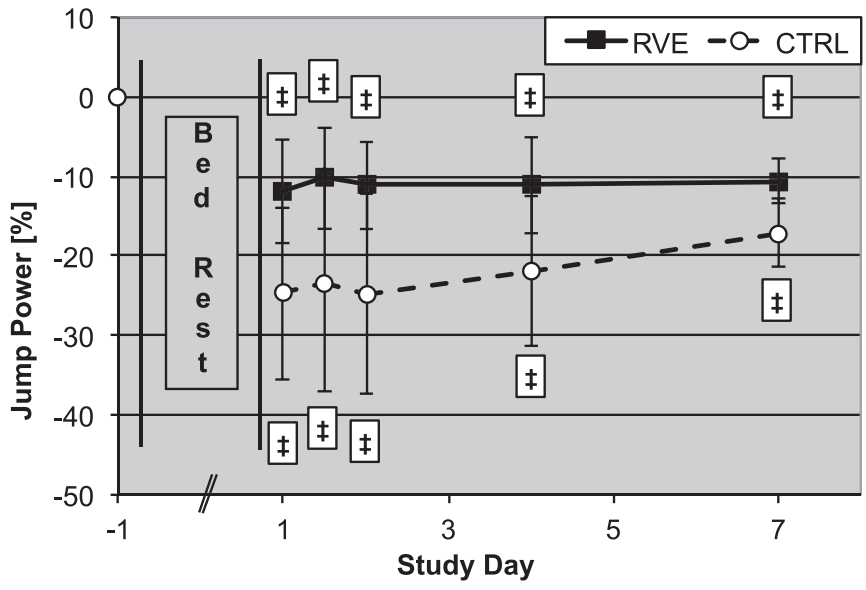

Fig. 4. Relative changes in peak jump power $\left(\mathrm{P}_{\text {peak }}\right)$ in both groups from BDC to $\mathrm{R}+7$. Immediately after bed rest $(\mathrm{R}+1 \mathrm{~m}) \mathrm{P}_{\text {peak }}$ decreased in both groups but to a larger extent in the control group. There were only small changes in the next 2 measurements $(\mathrm{R} 1+\mathrm{e}$ and $\mathrm{R}+2)$. The recovery of $\mathrm{P}_{\text {peak }}$ only occurred in the control group but not the RVE group in the week after bed rest. The repeated measures analysis showed a significant difference between the groups $(P=0.007)$. Changes within each group compared with baseline are represented by $* P<0.05$; $\uparrow P<0.01$; and $\ddagger P<0.001$. Error bars represent SD. Study day -1 represents BDC. Days 1 and 1.5 represent measurements in the $\mathrm{R}+1 \mathrm{~m}$ and $\mathrm{R}+1 \mathrm{e}$, respectively. Accordingly, study days 2, 4, and 7 represent $\mathrm{R}+2, \mathrm{R}+4$, and $\mathrm{R}+7$, respectively.

crease of $20 \%$, which trended toward significance. RVE preserved plantar-flexor muscle force, at least in the first moments after reambulation and the EMG-force relationship but only partially ameliorated the decline in muscle power to $12 \%$. RVE did not safeguard against the pronounced decline in plantarflexor muscle force that occurred between $\mathrm{R}+1 \mathrm{~m}$ and the $\mathrm{R}+1 \mathrm{e}, \mathrm{R}+2$ testing sessions. Interestingly, also, after $\mathrm{R}+1 \mathrm{~m}$, the marked decline in plantar-flexor muscle force was not associated with reductions in jump height or power.

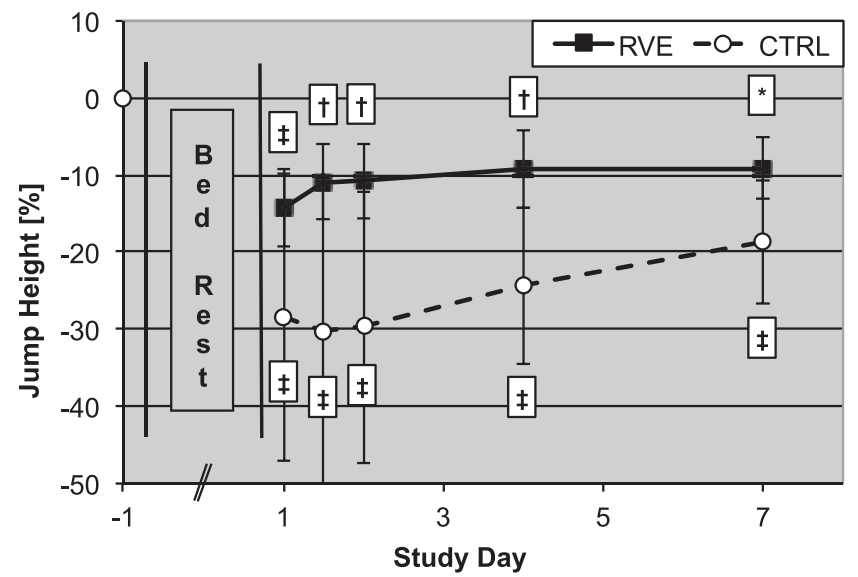

Fig. 5. Relative changes in jump height $\left(\mathrm{H}_{\mathrm{jump}}\right)$ in both groups from BDC to $\mathrm{R}+7$. Similar to $\mathrm{P}_{\text {peak }}, \mathrm{H}_{\text {jump }}$ declined from $\mathrm{BDC}$ to $\mathrm{R}+1 \mathrm{~m}$ and then did not change significantly on $\mathrm{R}+1 \mathrm{e}$ and $\mathrm{R}+2 . \mathrm{H}_{\text {jump }}$ also improved on $\mathrm{R}+4$ in the control group, whereas it remained unchanged in the RVE group. There was a significant difference between groups in repeated measures analysis $(P=$ $0.039)$. Changes within each group compared with baseline are represented by $* P<0.05 ; \dagger P<0.01$; and $\ddagger P<0.001$. Error bars represent SD. Study day -1 represents BDC. Days 1 and 1.5 represent measurements in the $\mathrm{R}+1 \mathrm{~m}$ and $\mathrm{R}+1 \mathrm{e}$, respectively. Accordingly, study days 2, 4, and 7 represent $\mathrm{R}+2, \mathrm{R}+4$, and $\mathrm{R}+7$, respectively.
Table 2. Correlations between jump parameters

\begin{tabular}{lccc}
\hline \hline & $\mathrm{H}_{\text {jump }}$ & $\mathrm{P}_{\text {peak }}$ & $\mathrm{P}_{\text {mean }}$ \\
\hline $\mathrm{H}_{\text {jump }}$ & & 0.82 & 0.34 \\
$\mathrm{P}_{\text {peak }}$ & 0.72 & & 0.60 \\
$\mathrm{P}_{\text {mean }}$ & 0.69 & 0.94 & \\
\hline
\end{tabular}

Top right (light-shaded) triangle: Spearman's correlation between absolute values at baseline data collection (BDC).

Bottom left (dark-shaded) triangle: Spearmans's partial correlations (controlling for study date) for percentage change in each parameter on recovery data collection immediately after the end of bed rest on the morning of reambulation compared with $\mathrm{BDC}$. With the exception of the correlation at BDC between maximal jump height $\left(\mathrm{H}_{\text {jump }}\right)$ and mean jump power $\left(\mathrm{P}_{\text {mean }}\right)$, all correlations were significant $(P \leq 0.005)$. $P_{\text {peak }}$, peak jump power.

To our knowledge, this study is the first study to measure jump power, height, and IPFF immediately after the end of bed rest and then again later on the same day. In the morning of $\mathrm{R}+1$, the participants were familiarized to orthostasis on a tilt table. This was done to ascertain stability of the cardiovascular system. Only after this were subjects asked to ambulate from the tilt table to the force plate of the countermovement jumps. Once the preparation for our experiments were finished, the volunteers first performed the countermovement jumps and then the isometric plantar-flexion contractions. All of this was done within the first hour of getting out of bed. The second session was done in the late afternoon or early evening after the participants had been walked for $45 \mathrm{~min}$ at a very gentle pace, but no other muscular testing was carried out. Our results indicate that there was a significant loss of isometric muscle force within 1 day. This not only happened in the control group but also in the training group. Possible explanations for this include muscle fatigue, development of pain, and loss of motivation. Other examinations in the same subjects (54) reported that participants of this study frequently complained of pain, especially in the lower extremity during the recovery phase, indicating that pain likely is a contributing factor. The development of muscle-fiber damage as part of reambulation (and testing) likely occurred after bed rest in association with the muscular pain reported by the subjects. This further "damage" to the musculature was potentially involved in the further deterioration of IPFF. Further evidence for muscle injury was provided by the marked increases in creatinine kinase levels in these subjects after reambulation (7). We did not have measures to assess motivation or muscle fatigue. We feel that this is a very important finding, as it emphasizes the importance of timing muscle-function tests during the recovery from bed rest and offers an additional and new explanation as to why results from muscle-function tests differ across studies. This study provides evidence that it is important to place the exams deemed most important by investigators early on the day of recovery, as the results are prone to be influenced by different factors if they are done later.

We have previously reported the changes in the absolute maximal values of the IPFF from BDC to $\mathrm{R}+1 \mathrm{~m}$ and $\mathrm{R}+1 \mathrm{e}$ elsewhere (12). The current findings extend this work in examining maximal and submaximal values and their relation to the EMG activity of the calf muscles. As expected, the absolute and averaged maximal values are similar and result in the same amount of changes in the two groups. The loss of $17 \%$ in maximally voluntary plantar-flexion force is similar to other publications. Loss of force or torque has been reported to 
range from $10 \%$ to $55 \%$, depending on the length of the study and the methodology used $(4,10,35,36,48,63)$. We did not find any changes in the EMG-RMS values during maximal plantar-flexion contractions in the control group when we compared $\mathrm{BDC}$ with $\mathrm{R}+1 \mathrm{~m}$. In the same time period, there was an increase in the \% RMS/\% force ratio of $20 \%$ with a trend toward significance in the control group, however. We used this ratio as a parameter for the neuromuscular drive. The observed increase suggested higher RMS values for the same amount of force, a decrease in neuromuscular drive. Results of other studies vary in their findings. In examining the same subjects, another research group did not find a decrease in EMG amplitude or maximal activation in the quadriceps musculature in the same study population $(44,45)$. Others have reported a loss of maximal EMG amplitude and neuromuscular drive $(4,26,34,36-38,63)$.

$\mathrm{P}_{\text {peak }}$ and $\mathrm{H}_{\text {jump }}$ decreased significantly in the control group. The observed declines of $28.5 \%$ and $24.1 \%$, respectively, are similar to those seen in other bed-rest studies showing an $\sim 25 \%$ decrease $(28,58)$. Although this work focused on muscle function, muscle size was also assessed in the BBR study. Data on changes of muscle volume of the different muscle groups of the lower extremity in the same study population have recently been published (8). They observed an $18.3 \%$ decrease in the calf muscles, $14.4 \%$ decrease in the quadriceps muscles, and $11.3 \%$ decrease in the hamstrings. The $18 \%$ loss in the calf muscles is similar to the $17 \%$ loss of plantar-flexion force but less than the $28.5 \%$ and $24.1 \%$ decrease in $\mathrm{P}_{\text {peak }}$ and $\mathrm{H}_{\text {jump. Although there is no doubt that }}$ muscle atrophy plays an important role in loss of jump power, it is possible that other factors also play a role. Changes in neuromuscular drive, although only shown with a trend to significance in the IPFF measurements, might be one of these factors. Moreover, the ability of muscle to store elastic energy, which is known to be essential for jumping performance (40), could potentially have been affected by bed rest. Data from astronauts suggest that maximal explosive maneuvers are greatly impaired after their return to Earth (5), and it therefore seems important to study whether there is deconditioning of elastic energy storage (in passive elements, such as connective tissue, and in active motor control of eccentric muscle loading) in future studies.

We found that the RVE training protocol was able to preserve IPFF, at least until immediately after reambulation. Other training modalities have shown similar results or at least were able to reduce the loss of force $(2-4,63)$. This makes the method equally effective in terms of preserving muscle force. The EMG-RMS values and the \% RMS-\% force ratio did not change in the RVE group, similar to the maximal isometric plantar flexions. The intervention arms of previously cited studies have also shown that there are not significant changes in EMG amplitude or neuromuscular drive when the training was effectively preventing loss of muscle force $(4,63)$.

Although the loss of jump power and height was smaller than in the control group, the RVE was not able to maintain this muscle function completely. There also was a decrease in muscle CSA in this group. In these exercise subjects, a $6.6 \%$ decline in the volume of the calf muscles, a 3.3\% decline in the quadriceps, and a $10.9 \%$ decline in the hamstrings were observed (9). The amount of muscle power loss during countermovement jumps was similar to results from a flywheel exer- cise group of a 90-day bed-rest study (58). In the same study, the population of calf muscle CSA decreased by $15 \%$, whereas thigh muscle CSA did not change (4).

Lastly, our observation that the magnitude in changes of $\mathrm{P}_{\text {peak }}$ and $\mathrm{P}_{\text {mean }}$ was similar and that there were significant correlations of the two parameters, along with $\mathrm{H}_{\text {jump }}$, suggests that although measuring somewhat different qualities of neuromuscular function, they all were quite uniformly affected by bed rest.

Summarizing the above, it appears that muscle mass, muscle force, and muscle power all decline in different degrees during bed rest and that countermeasures vary in their ability to prevent these losses. The incomplete maintenance of IPFF in the RVE group, despite maintenance of jump power and also the diverging time course of recovery of IPFF (further deterioration before recovery) and jump power (maintenance and then recovery), requires further attention. These observations clearly show that muscle force during a static contraction and jumping performance assesses two distinct qualities. Apparently, the factors that led to deterioration of muscle force within the observation interval did not seem to grossly affect jumping performance. Conversely, the improvements in plantar-flexion force that occurred between days $R+2$ and $R+7$ in the RVE group (Fig. 2) did not translate into a noticeable improvement of jumping performance (Fig. 4). This and the fact that a musculature, which was seemingly well preserved during 56 days of bed rest by RVE, deteriorated very considerably within $8-10 \mathrm{~h}$ after reambulation, probably triggered by a 45-min walk at gentle pace, demonstrate that there is a muscle "quality", which so far, has escaped our attention. There are, no doubt, a number of differences between isometric plantar flexion and jump maneuvers, which could play a role in the differential changes that we observed after bed rest. In terms of motor control, maintenance of muscle activity during isometric muscle contractions is in part dependent on feedback from afferent motoneurons (31), whereas jumping maneuvers involve ballistic/explosive muscle contraction, during which there is no time for modulation of muscle activity by sensory feedback signals (24). Also, energy-storing capacity, i.e., the muscle's ability to act as a spring, but also energy-dissipating capacity, i.e., the ability of the muscle to act as a break (20), and changes in tendon compliance $(39,41,51)$, as well as a number of other mechanisms, including changes in the forcevelocity curves, could well play a role.

In comparison with studying the effect of bed rest, comparatively little work has examined the time course of recovery of neuromuscular function after prolonged bed rest. The parameters measured in the current study only recovered partially in the first week after bed rest. Measurements on jump power and height after bed rest (58) and explosive maneuvers after spaceflight (5) suggest that the recovery of these parameters would take a number of weeks to months, depending on parameter and length of bed rest or space travel. Although we could not identify work on the recovery of plantar-flexion isometric force after bed rest, the recovery of maximal knee-extension force after limb suspension appears to occur within $1 \mathrm{wk}$ (11). The time course of recovery will, no doubt, depend on the extent of loss, and data from patients immobilized for $7 \mathrm{wk}$ after ankle fracture showed recovery of isometric plantar extension force between 5 and $10 \mathrm{wk}$ of rehabilitation (65). More precise 
evaluation of the time course of recovery of such neuromuscular parameters would be of interest in future work.

Finally, it needs to be emphasized that the importance of muscle power also has become evident in recent studies in older adults, who showed muscle power to better correlate with quality of life, activities of daily living, morbidity, and even mortality than with muscle force $(6,16,22,33,62)$. This suggests that it will be important not only to include the assessment of muscle power in routine muscle-function measurements but also to design training methods that are able to prevent both muscle force and power.

Although this study brings new data into the field, it also has limitations. As in almost all studies in real or simulated microgravity, the sample size in this study is also small, which may have prevented some effects from reaching statistical significance. Also, extrapolation to wider populations should be made with caution for the same reason, but this may also be a factor in explaining why studies in bed rest have found such a variation in changes of plantar-flexion force $(4,10,35,36$, $48,63)$. It is also important to note that further study should examine the effect of vibration alone or resistive exercise alone on the parameters measured here. Due to cost limitations in bed-rest studies, the BBR study aimed first to evaluate the effectiveness of the countermeasure program as a whole, with further studies to be planned if the countermeasure proves effective. Also, future work could consider whether additional benefits might be gained by other forms of exercise (such as explosive plantar flexion vs. high load-resistive exercise) on preventing loss in plantar-flexor isometric force. Finally, it is evident that a longer follow-up would be required to study the completeness of recovery.

In conclusion, we found that muscle force declines within the first day of recovery from bed rest. Furthermore, we reported a larger loss of jump power than of isometric muscle force in both the control and training group. This study's findings are similar to other studies in regard to loss of IPFF. With this decline, we also found a change in neuromuscular drive. RVE was able to preserve IPFF but only attenuates the loss of jump power and height. Remarkably, jump power and height were maintained in continued tests after initial postbedrest measurements, despite continued deterioration in IPFF. Future studies should routinely include tests not only for muscle force but also power and find countermeasures that prevent the loss both muscle functions.

\section{ACKNOWLEDGMENTS}

We thank all study participants for their selfless contribution and commitment; without them, the success of this study would not have been possible. Additionally, we express our gratefulness to the Department of Radiology of the Charité-Campus Benjamin Franklin, the Centre for Muscle and Bone Research, and last but not least, the staff on Ward 18 for their wonderful support.

\section{GRANTS}

The BBR study was funded by Grant 14431/02/NL/SH2 from the European Space Agency. It was further supported by Charité-University Medicine Berlin (Campus Benjamin Franklin), DLR (German AeroSpace), Novotec Medical, MSD Sharp \& Dohme, Lilly Germany, Servier Germany, Hoffmann-LaRoche, Siemens, Novartis, and Seca.

\section{DISCLOSURES}

No conflicts of interest, financial or otherwise, are declared by the author(s).

\section{REFERENCES}

1. Adams GR, Caiozzo VJ, Baldwin KM. Skeletal muscle unweighting: spaceflight and ground-based models. J Appl Physiol 95: 2185-2201, 2003.

2. Akima H, Kubo K, Kanehisa H, Suzuki Y, Gunji A, Fukunaga T. Leg-press resistance training during 20 days of 6 degrees head-down-tilt bed rest prevents muscle deconditioning. Eur J Appl Physiol 82: 30-38, 2000.

3. Akima H, Ushiyama J, Kubo J, Tonosaki S, Itoh M, Kawakami Y, Fukuoka H, Kanehisa H, Fukunaga T. Resistance training during unweighting maintains muscle size and function in human calf. Med Sci Sports Exerc 35: 655-662, 2003.

4. Alkner BA, Tesch PA. Knee extensor and plantar flexor muscle size and function following 90 days of bed rest with or without resistance exercise. Eur J Appl Physiol 93: 294-305, 2004.

5. Antonutto G, Capelli C, Girardis M, Zamparo P, di Prampero PE. Effects of microgravity on maximal power of lower limbs during very short efforts in humans. J Appl Physiol 86: 85-92, 1999.

6. Bean JF, Leveille SG, Kiely DK, Bandinelli S, Guralnik JM, Ferrucci L. A comparison of leg power and leg strength within the InCHIANTI study: which influences mobility more? J Gerontol A Biol Sci Med Sci 58: $728-733,2003$.

7. Belavý D, Seibel M, Roth H, Armbrecht G, Rittweger J, Felsenberg D. The effects of bed rest and countermeasure exercise on the endocrine system in male adults-evidence for immobilization induced reduction in SHBG levels. J Endocrinol Invest. doi:10.3275/7606.

8. Belavý DL, Miokovic T, Armbrecht G, Richardson CA, Rittweger J, Felsenberg D. Differential atrophy of the lower-limb musculature during prolonged bed-rest. Eur J Appl Physiol 107: 489-499, 2009.

9. Belavý DL, Miokovic T, Armbrecht G, Rittweger J, Felsenberg D. Resistive vibration exercise reduces lower limb muscle atrophy during 56-day bed-rest. J Musculoskelet Neuronal Interact 9: 225-235, 2009.

10. Berg HE, Larsson L, Tesch PA. Lower limb skeletal muscle function after 6 wk of bed rest. J Appl Physiol 82: 182-188, 1997.

11. Berg HE, Tesch PA. Changes in muscle function in response to 10 days of lower limb unloading in humans. Acta Physiol Scand 157: 63-70, 1996.

12. Blottner D, Salanova M, Puttmann B, Schiffl G, Felsenberg D, Buehring B, Rittweger J. Human skeletal muscle structure and function preserved by vibration muscle exercise following 55 days of bed rest. Eur J Appl Physiol 97: 261-271, 2006.

13. Bogaerts A, Delecluse C, Claessens AL, Coudyzer W, Boonen S, Verschueren SM. Impact of whole-body vibration training versus fitness training on muscle strength and muscle mass in older men: a 1-year randomized controlled trial. J Gerontol A Biol Sci Med Sci 62: 630-635, 2007.

14. Cardinale M, Rittweger J. Vibration exercise makes your muscles and bones stronger: fact or fiction? J Br Menopause Soc 12: 12-18, 2006.

15. Cavagna GA. Force platforms as ergometers. J Appl Physiol 39: 174179, 1975.

16. Cesari M, Pahor M, Lauretani F, Zamboni V, Bandinelli S, Bernabei R, Guralnik JM, Ferrucci L. Skeletal muscle and mortality results from the InCHIANTI Study. J Gerontol A Biol Sci Med Sci 64: 377-384, 2009.

17. Chopard A, Arrighi N, Carnino A, Marini JF. Changes in dysferlin, proteins from dystrophin glycoprotein complex, costameres, and cytoskeleton in human soleus and vastus lateralis muscles after a long-term bedrest with or without exercise. FASEB J 19: 1722-1724, 2005.

18. Cochrane DJ, Sartor F, Winwood K, Stannard SR, Narici MV, Rittweger J. A comparison of the physiologic effects of acute whole-body vibration exercise in young and older people. Arch Phys Med Rehabil 89: $815-821,2008$.

19. Cochrane DJ, Stannard SR. Acute whole body vibration training increases vertical jump and flexibility performance in elite female field hockey players. Br J Sports Med 39: 860-865, 2005.

20. Cochrane DJ, Stannard SR, Sargeant AJ, Rittweger J. The rate of muscle temperature increase during acute whole-body vibration exercise. Eur J Appl Physiol 103: 441-448, 2008.

21. Convertino VA, Bloomfield SA, Greenleaf JE. An overview of the issues: physiological effects of bed rest and restricted physical activity. Med Sci Sports Exerc 29: 187-190, 1997.

22. Cuoco A, Callahan DM, Sayers S, Frontera WR, Bean J, Fielding RA. Impact of muscle power and force on gait speed in disabled older men and women. J Gerontol A Biol Sci Med Sci 59: 1200-1206, 2004.

23. Davies CT, Rennie R. Human power output. Nature 217: 770-771, 1968. 
24. Desmedt JE, Godaux E. Ballistic contractions in man: characteristic recruitment pattern of single motor units of the tibialis anterior muscle. $J$ Physiol 264: 673-693, 1977.

25. di Prampero PE, Narici MV. Muscles in microgravity: from fibres to human motion. J Biomech 36: 403-412, 2003.

26. Duchateau J. Bed rest induces neural and contractile adaptations in triceps surae. Med Sci Sports Exerc 27: 1581-1589, 1995.

27. Edgerton VR, McCall GE, Hodgson JA, Gotto J, Goulet C, Fleischmann K, Roy RR. Sensorimotor adaptations to microgravity in humans. J Exp Biol 204: 3217-3224, 2001.

28. Ferretti G, Berg HE, Minetti AE, Moia C, Rampichini S, Narici MV. Maximal instantaneous muscular power after prolonged bed rest in humans. J Appl Physiol 90: 431-435, 2001.

29. Fitts RH, Riley DR, Widrick JJ. Physiology of a microgravity environment invited review: microgravity and skeletal muscle. J Appl Physiol 89: 823-839, 2000.

30. Fitts RH, Trappe SW, Costill DL, Gallagher PM, Creer AC, Colloton PA, Peters JR, Romatowski JG, Bain JL, Riley DA. Prolonged space flight-induced alterations in the structure and function of human skeletal muscle fibres. J Physiol 588: 3567-3592, 2010.

31. Gandevia SC, Macefield G, Burke D, McKenzie DK. Voluntary activation of human motor axons in the absence of muscle afferent feedback. The control of the deafferented hand. Brain 113: 1563-1581, 1990.

32. Issurin VB. Vibrations and their applications in sport. A review. J Sports Med Phys Fitness 45: 324-336, 2005.

33. Katula JA, Rejeski WJ, Marsh AP. Enhancing quality of life in older adults: a comparison of muscular strength and power training. Health Qual Life Outcomes 6: 45, 2008.

34. Kawakami Y, Akima H, Kubo K, Muraoka Y, Hasegawa H, Kouzaki M, Imai M, Suzuki Y, Gunji A, Kanehisa H, Fukunaga T. Changes in muscle size, architecture, and neural activation after 20 days of bed rest with and without resistance exercise. Eur J Appl Physiol 84: 7-12, 2001.

35. Koryak Y. Contractile properties of the human triceps surae muscle during simulated weightlessness. Eur J Appl Physiol Occup Physiol 70: 344-350, 1995.

36. Koryak Y. Electromyographic study of the contractile and electrical properties of the human triceps surae muscle in a simulated microgravity environment. J Physiol 510: 287-295, 1998.

37. Koryak YU. Electrically evoked and voluntary properties of the human triceps surae muscle: effects of long-term spaceflights. Acta Physiol Pharmacol Bulg 26: 21-27, 2001.

38. Kozlovskaya IB, Kreidich Yu V, Oganov VS, Koserenko OP. Pathophysiology of motor functions in prolonged manned space flights. Acta Astronaut 8: 1059-1072, 1981.

39. Kubo K, Akima H, Ushiyama J, Tabata I, Fukuoka H, Kanehisa H, Fukunaga T. Effects of resistance training during bed rest on the viscoelastic properties of tendon structures in the lower limb. Scand J Med Sci Sports 14: 296-302, 2004.

40. Kurokawa S, Fukunaga T, Fukashiro S. Behavior of fascicles and tendinous structures of human gastrocnemius during vertical jumping. $J$ Appl Physiol 90: 1349-1358, 2001.

41. Lambertz D, Perot C, Kaspranski R, Goubel F. Effects of long-term spaceflight on mechanical properties of muscles in humans. J Appl Physiol 90: 179-188, 2001.

42. Mischi M, Kaashoek I. Electromyographic hyperactivation of skeletal muscles by time-modulated mechanical stimulation. Conf Proc IEEE Eng Med Biol Soc 2007: 5373-5376, 2007.

43. Mosley JR, Lanyon LE. Strain rate as a controlling influence on adaptive modeling in response to dynamic loading of the ulna in growing male rats. Bone 23: 313-318, 1998.

44. Mulder ER, Kuebler WM, Gerrits KH, Rittweger J, Felsenberg D, Stegeman DF, de Haan A. Knee extensor fatigability after bedrest for 8 weeks with and without countermeasure. Muscle Nerve 36: 798-806, 2007.

45. Mulder ER, Stegeman DF, Gerrits KH, Paalman MI, Rittweger J, Felsenberg D, de Haan A. Strength, size and activation of knee extensors followed during 8 weeks of horizontal bed rest and the influence of a countermeasure. Eur J Appl Physiol 97: 706-715, 2006.

46. NASA. Bioastronautics Roadmap (Online). http://bioastroroadmap.nasa. gov/index.jsp. Accessed 3 November 2010.

47. Pavy-Le Traon A, Heer M, Narici MV, Rittweger J, Vernikos J. From space to Earth: advances in human physiology from 20 years of bed rest studies (1986-2006). Eur J Appl Physiol 101: 143-194, 2007.
48. Portero P, Vanhoutte C, Goubel F. Surface electromyogram power spectrum changes in human leg muscles following 4 weeks of simulated microgravity. Eur J Appl Physiol Occup Physiol 73: 340-345, 1996.

49. Prou E, Marini JF. Muscle research in space-increased muscle susceptibility to exercise-induced damage after a prolonged bedrest. Int J Sports Med 18, Suppl 4: S317-S320, 1997.

50. Qin YX, Rubin CT, McLeod KJ. Nonlinear dependence of loading intensity and cycle number in the maintenance of bone mass and morphology. J Orthop Res 16: 482-489, 1998.

51. Reeves ND, Maganaris CN, Ferretti G, Narici MV. Influence of 90-day simulated microgravity on human tendon mechanical properties and the effect of resistive countermeasures. J Appl Physiol 98: 2278-2286, 2005.

52. Ribot-Ciscar E, Butler JE, Thomas CK. Facilitation of triceps brachii muscle contraction by tendon vibration after chronic cervical spinal cord injury. J Appl Physiol 94: 2358-2367, 2003.

53. Rittweger J. Vibration as an exercise modality: how it may work, and what its potential might be. Eur J Appl Physiol 108: 877-904, 2010.

54. Rittweger J, Belavý D, Hunek P, Gast U, Boerst H, Feilcke B, Armbrecht G, Mulder E, Schubert H, Richardson C, de Haan A, Stegeman DF, Schiessl H, Felsenberg D. Highly demanding resistive vibration exercise program is tolerated during 56 days of strict bed-rest. Int J Sports Med 27: 553-559, 2006.

55. Rittweger J, Beller G, Armbrecht G, Mulder E, Buehring B, Gast U, Dimeo F, Schubert H, de Haan A, Stegeman DF, Schiessl H, Felsenberg D. Prevention of bone loss during 56 days of strict bed rest by side-alternating resistive vibration exercise. Bone 46: 137-147, 2010.

56. Rittweger J, Beller G, Felsenberg D. Acute physiological effects of exhaustive whole-body vibration exercise in man. Clin Physiol 20: $134-$ 142, 2000.

57. Rittweger J, Ehrig J, Just K, Mutschelknauss M, Kirsch KA, Felsenberg $\mathbf{D}$. Oxygen uptake in whole-body vibration exercise: influence of vibration frequency, amplitude, and external load. Int J Sports Med 23: 428-432, 2002.

58. Rittweger J, Felsenberg D, Maganaris C, Ferretti JL. Vertical jump performance after 90 days bed rest with and without flywheel resistive exercise, including a 180 days follow-up. Eur J Appl Physiol 100: 427-436, 2007

59. Rittweger J, Schiessl H, Felsenberg D, Runge M. Reproducibility of the jumping mechanography as a test of mechanical power output in physically competent adult and elderly subjects. J Am Geriatr Soc 52: 128-131, 2004.

60. Roelants M, Verschueren SM, Delecluse C, Levin O, Stijnen V. Whole-body-vibration-induced increase in leg muscle activity during different squat exercises. J Strength Cond Res 20: 124-129, 2006.

61. Roll JP, Vedel JP, Ribot E. Alteration of proprioceptive messages induced by tendon vibration in man: a microneurographic study. Exp Brain Res 76: 213-222, 1989.

62. Runge M, Rittweger J, Russo CR, Schiessl H, Felsenberg D. Is muscle power output a key factor in the age-related decline in physical performance? A comparison of muscle cross section, chair-rising test and jumping power. Clin Physiol Funct Imaging 24: 335-340, 2004.

63. Schulze K, Gallagher P, Trappe S. Resistance training preserves skeletal muscle function during unloading in humans. Med Sci Sports Exerc 34: 303-313, 2002.

64. Srihari T, Seedorf U, Pette D. Ipsi-and contralateral changes in rabbit soleus myosins by cross-reinnervation. Pflügers Arch 390: 246-249, 1981.

65. Stevens JE, Pathare NC, Tillman SM, Scarborough MT, Gibbs CP, Shah P, Jayaraman A, Walter GA, Vandenborne K. Relative contributions of muscle activation and muscle size to plantarflexor torque during rehabilitation after immobilization. J Orthop Res 24: 1729-1736, 2006.

66. Trappe S, Costill D, Gallagher P, Creer A, Peters JR, Evans H, Riley DA, Fitts RH. Exercise in space: human skeletal muscle after 6 months aboard the International Space Station. J Appl Physiol 106: 1159-1168, 2009.

67. Trappe SW, Trappe TA, Lee GA, Widrick JJ, Costill DL, Fitts RH. Comparison of a space shuttle flight (STS-78) and bed rest on human muscle function. J Appl Physiol 91: 57-64, 2001.

68. Trappe TA, Burd NA, Louis ES, Lee GA, Trappe SW. Influence of concurrent exercise or nutrition countermeasures on thigh and calf muscle size and function during 60 days of bed rest in women. Acta Physiol (Oxf) 191: 147-159, 2007 